\title{
Multidimensional predictors of negative symptoms in antipsychotic-naive first-episode psychosis
}

\author{
Alba Toll, MD; Laura Blanco-Hinojo, PhD; Daniel Bergé, PhD; Xavier Duran, PhD; \\ Irene Canosa, MHN; Teresa Legido, PhD; Federico Marmol, PhD; Víctor Pérez-Solà, PhD; \\ Emilio Fernández-Egea, PhD; Anna Mané, PhD
}

\begin{abstract}
Background: Despite a large body of schizophrenia research, we still have no reliable predictors to guide treatment from illness onset. The present study aimed to identify baseline clinical or neurobiological factors — including peripheral brain-derived neurotrophic factor (BDNF) levels and amygdala or hippocampal relative volumes - that could predict negative symptomatology and persistent negative symptoms in first-episode psychosis after 1 year of follow-up. Methods: We recruited 50 drug-naive patients with first-episode psychosis and 50 age- and sex-matched healthy controls to study brain volumes. We performed univariate and multiple and logistic regression analyses to determine the association between baseline clinical and neurobiological variables, score on the PANSS negative subscale and persistent negative symptoms after 1 year of follow-up. Results: Low baseline serum BDNF levels $(p=0.011)$, decreased left amygdala relative volume $(p=0.001)$ and more severe negative symptomatology $(p=0.021)$ predicted the severity of negative symptoms at 1 year, as measured by the PANSS negative subscale. Low baseline serum BDNF levels $(p=0.012)$ and decreased left amygdala relative volume $(p=0.010)$ predicted persistent negative symptoms at 1 year. Limitations: We were unable to assess negative symptoms and their dimensions with next-generation scales, which were not available when the study was initiated. Conclusion: This study shows that a set of variables at baseline, including low BDNF levels, smaller left amygdala relative volume and score on the PANSS negative subscale are significant predictors of outcomes in first-episode psychosis. These findings might offer an initial step for tailoring treatments in first-episode psychosis.
\end{abstract}

\section{Introduction}

Schizophrenia is a chronic, lifelong, disabling disorder that develops via complex, heterogeneous, multifactorial (genetic, developmental and environmental) pathways. ${ }^{1}$ It is 1 of the top 15 leading causes of disability worldwide, ${ }^{2}$ impairing critical areas of everyday functioning. ${ }^{3}$ Although early interventions may improve treatment outcomes, ${ }^{4}$ there is a notable lack of reliable predictors to help guide an objective, datadriven personalized approach to treatment after illness onset. ${ }^{5}$

Given the complexity of schizophrenia, it is essential to identify unambiguous and distinctive predictors of outcomes, ${ }^{6}$ such as negative symptoms. ${ }^{7}$ Negative symptoms are among the most important determinants of functioning 8,9 in schizophrenia. They can be present at any stage of the disorder, even in the early stages, with prevalence in up to $50 \%$ of patients. ${ }^{10}$ Longitudinal studies in early stages have found that when these symptoms are present at baseline, they tend to be persistent, although severity may vary over time.
Therefore, it would be highly valuable to identify - at or near disease onset - those patients who are most likely to develop severe negative symptoms; this would facilitate the early implementation of personalized interventions. ${ }^{11}$

There are different approaches to measuring negative symptoms. The most common approach is to rate all negative symptoms without considering the possible cause (e.g., positive symptoms, adverse effects, social deprivation or others); PANSS is one of the most widely used scales for this purpose. Another approach is to restrict negative symptoms to those considered primary or deficit symptoms ${ }^{12,13}$ after ruling out secondary causes. An alternative approach to studying negative symptoms is to use persistent negative symptoms; these may include secondary negative symptoms, but only those that have failed to respond to the usual treatments and are evident for more than 6 months. Persistent negative symptoms are also highly relevant because they lead to functional impairment and represent an unmet therapeutic need. ${ }^{14}$

Correspondence to: L. Blanco, MRI Department, Parc de Salut Mar, Passeig Marítim 25-29 08003, Barcelona, Spain; laura.blanco02@gmail.com

Submitted Aug. 7, 2021; Revised Oct. 15, 2021; Accepted Nov. 1, 2021

Cite as: J Psychiatry Neurosci 2022 January 19;47(1). doi: 10.1503/jpn.210138 
Several clinical factors have been linked to negative symptoms in general. For example, more severe negative symptoms have been observed in males, ${ }^{15,16}$ in patients who developed schizophrenia at a younger age $\mathrm{e}^{17,18}$ and in those with a longer duration of untreated psychosis. ${ }^{19,20}$ However, the association between negative symptoms and these clinical factors has not been consistent in the studies to date. ${ }^{15,19,21}$ The influence of cannabis use on negative symptomatology is poorly understood, but a recent meta-analysis showed that recently abstinent patients showed negative symptoms that were less severe than nonusers. ${ }^{22}$ In addition to these clinical markers, a considerable amount of research has been conducted to identify neurobiological factors that could predict outcomes and help to select optimal treatment strategies. ${ }^{5}$

Morphological and functional brain alterations have been widely reported in schizophrenia. ${ }^{23}$ Structural and functional changes in the limbic system and medial temporal lobe are central to the symptomatology associated with psychosis, ${ }^{24}$ and smaller relative hippocampal volumes are commonly observed in patients with first-episode psychosis and schizophrenia. ${ }^{25}$ Furthermore, these hippocampal abnormalities are associated with the emergence of negative symptoms and persistent negative symptoms in patients with schizophrenia and first-episode psychosis. ${ }^{10,26}$ Smaller amygdala volumes have also been reported in patients with schizophrenia and first-episode psychosis compared to controls. ${ }^{27,28}$ In addition, volume alterations in the amygdala have been associated with both negative symptoms and persistent negative symptoms in schizophrenia and in firstepisode psychosis during follow-up. ${ }^{10,26}$

Brain-derived neurotrophic factor (BDNF) has been implicated in the pathophysiology of schizophrenia, and it has been shown to play a crucial role in neuronal development, transmission, regulation and synaptic plasticity. ${ }^{29}$ Data from systematic reviews and meta-analyses show that patients with schizophrenia ${ }^{30}$ and first-episode psychosis $^{31}$ have lower peripheral BDNF levels than healthy controls. Crosssectional studies have found an association between peripheral BDNF levels and the clinical features of schizophrenia, such as the severity of negative symptoms. ${ }^{32}$ These data suggest that peripheral BDNF levels could be a neurobiological predictor of psychosis and psychosis phenotypes. However, more longitudinal studies are needed in firstepisode psychosis to better determine the association between peripheral BDNF levels and different outcome parameters, including negative symptoms.

In summary, there is a clear need to identify predictors of outcomes in schizophrenia and guide individualized treatment strategies from illness onset. Negative symptoms are distinctive features of schizophrenia and critical determinants of outcomes. Although various sociodemographic, clinical and neurobiological factors have been associated with negative symptoms, few studies have evaluated the predictive value of these factors longitudinally starting from disease onset, and even fewer have considered the sources of secondary negative symptoms or persistent negative symptoms. Finally, to our knowledge, no studies have assessed all 3 factors (clinical, neuroimaging and biochemical) together.
The primary objective of the present study was to determine whether baseline clinical and neurobiological factors including duration of untreated psychosis, clinical symptoms, cannabis use, peripheral BDNF levels and amygdala or hippocampal relative volumes - could predict negative symptomatology and persistent negative symptoms at 1 year of follow-up in patients with first-episode psychosis. A second aim was to measure and compare hippocampal and amygdalar volumes in patients with first-episode psychosis and healthy controls to determine significant differences.

We hypothesized that this combination of clinical and neurobiological parameters would predict negative symptoms and persistent negative symptoms at 1-year follow-up. We also hypothesized that patients with first-episode psychosis would have smaller hippocampal and amygdalar relative volumes than healthy controls.

\section{Methods}

\section{Study population}

Fifty consecutive, drug-naive patients with first-episode psychosis who were treated between April 2013 and July 2017 at the Estudi $i$ Tractament de Primers Episodis Psicotics (ETEP) Program at Hospital del Mar (Barcelona, Spain) were included in this study. The ETEP program is a specialized early intervention service for people aged 18 to 35 who experience first-episode psychosis. It provides multimodal intervention, including extensive assessment and intensive medical and psychosocial treatment (for more details, see Bergé and colleagues ${ }^{33}$ ).

For the present study, inclusion criteria for patients were as follows: age 18 to 35 years; fulfillment of DSM-IV-TR criteria for brief psychotic disorder, schizophreniform disorder, schizophrenia with less than 1 year of symptoms or unspecified psychosis; no previous history of severe neurologic medical conditions or severe traumatic brain injury; presumed IQ above 80 based on clinical records (evidence from previous IQ assessments or suggested by the patient's educational or employment level); and no substance abuse or dependence disorders, except for cannabis or nicotine use. All patients were antipsychoticand antidepressant-naive at inclusion. Treatment with benzodiazepines was allowed. We excluded affective psychosis to homogenize the sample, because negative symptoms in affective psychosis can differ in terms of whether they are primary or secondary and persistent or transient ${ }^{34}$ (see Appendix 1, Figure S1 and Table S1, available at www.jpn.ca/lookup/ doi/10.1503/jpn.210138/tab-related-content, for detailed information on patients excluded from the neuroimaging study).

We used 50 age- and sex-matched healthy volunteers as a control group for anatomic measurements. All healthy controls underwent a complete medical interview. Those with relevant medical or neurologic disorders, substance abuse disorders or psychiatric illnesses were excluded from the study. None of the healthy controls was undergoing medical treatment at study inclusion. Given the characteristics of the study design, we used no statistical methods to predetermine sample sizes.

The local ethics committee approved this study, and all participants provided written informed consent. 


\section{Clinical assessment and demographic data}

All patients underwent a comprehensive assessment at baseline and 1-year follow-up, performed by 2 experienced psychiatrists (A.M., D.B.). The assessment included sociodemographic variables; the Structured Clinical Interview for DSM-IV-TR Axis I disorders for diagnosis; an assessment of substance use, including tobacco (cigarettes per day) and cannabis use (joints per week, after dichotomization between user/nonuser); the Positive and Negative Syndrome Scale (PANSS) for symptoms related to psychosis; ${ }^{35}$ the Global Assessment of Functioning (GAF) ${ }^{36}$ for functionality; and the Calgary Depression Scale for Schizophrenia (CDSS) for depressive symptoms. ${ }^{37} \mathrm{We}$ collected patients' antipsychotic dose at 1-year follow-up from their medical records. We converted doses of antipsychotic medications to chlorpromazine equivalents $(\mathrm{mg} / \mathrm{d})$ to facilitate comparisons. ${ }^{38}$

To minimize the effect of secondary negative symptom sources, we excluded all patients who scored 6 or higher on the CDSS at baseline or at follow-up. ${ }^{39} \mathrm{We}$ also included antipsychotic treatments at follow-up in the analyses.

We also assessed for the presence of persistent negative symptoms, defined as 1 or more negative symptoms of moderate or higher severity (PANSS negative subscale $>3$ ), not confounded by depression (CDSS $<6$ ) or parkinsonism at baseline and after 1 year of treatment. ${ }^{39}$

\section{Collection of blood samples and determination of serum BDNF levels in patients at baseline}

We obtained baseline fasting blood samples upon patients' arrival at the health centre before administering any medication (except for benzodiazepines). All blood samples were obtained in the morning (between $8 \mathrm{am}$ and $12 \mathrm{pm}$ ) to avoid circadian fluctuations in BDNF levels, which have been reported to occur in men but not in women..$^{40}$ Blood samples were collected in glass K3-EDTA blood-drawing tubes for whole blood. Serum was isolated by centrifugation at $300 \times g$ for 15 minutes, and then removed and stored at $-80^{\circ} \mathrm{C}$ until analysis.

BDNF levels were measured using a sandwich enzymelinked immunoabsorbent assay kit according to the manufacturer's instructions (ChemiKine; Chemicon). Absorbencies were determined using the Wallac Victor 2 microplate reader, with the wavelength set at $450 \mathrm{~nm}$. BDNF concentrations were detected according to the standard curve, which was constructed from duplicate samples containing appropriate concentrations. All samples were analyzed in duplicate. The calculated overall intra- and interassay variation coefficients were $3.7 \%$ and $8.5 \%$, respectively. The detection limit of the BDNF assay was $15 \mathrm{pg} / \mathrm{mL}$.

\section{Image acquisition and processing at baseline}

Brain imaging was performed with a Philips Achieva $3.0 \mathrm{~T}$ MRI scanner (Philips Healthcare) equipped with an 8-channel phased-array head coil. The imaging protocol involved the acquisition of high-resolution anatomic 3-dimensional images based on a $T_{1}$-weighted fast spoiled gradient inversion recovery prepared sequence with the following parameters: repetition time $8.2 \mathrm{~ms}$; echo time $3.8 \mathrm{~ms}$; flip angle $8^{\circ}$; field of view $24 \mathrm{~cm}$; matrix $256 \times 256$ pixels; in-plane resolution $0.94 \times 0.94 \mathrm{~mm}^{2}$; and slice thickness $1 \mathrm{~mm}$.

Anatomic images were visually inspected before analysis by a trained operator to detect any motion effect. No gross brain abnormalities were identified. The quality of the raw $T_{1}$-weighted MRI scans was quantitatively assessed using the automated weighted image quality rating (a measure of general image quality that combines the parameters of noise, inhomogeneities and spatial resolution) in the CAT12 toolbox (http://dbm.neuro.uni-jena.de/ cat/); a score of more than $60 \%$ is considered sufficient quality for inclusion in subsequent analyses. All scans passed this threshold.

We performed volumetric segmentation using the fully automated and validated segmentation software package FreeSurfer v6.0 (http:/ / surfer.nmr.mgh.harvard.edu) with the default "recon-all" stream, as described in previous studies. ${ }^{41-44}$ Briefly, important preprocessing steps included removal of non-brain tissue, intensity normalization, automated Talairach transformation and segmentation of the subcortical white matter and deep grey matter volumetric structures. ${ }^{45}$ Segmentations of the hippocampus and amygdala (left and right hemisphere) were visually inspected for accuracy by overlaying the segmentation label of each structure on the individual $T_{1}$-weighted brain scan. Specifically, we used the imaging quality control protocol for subcortical segmentations developed by the ENIGMA consortium (http:/ / enigma.ini.usc.edu/protocols/imaging-protocols/) to generate a standard set of images from every participant's brain scan, displaying boundaries of segmented structures on a series of slices in the axial, coronal and sagittal planes (Figure 1) for visual inspection of segmentation inaccuracies. For each participant, we extracted estimated hippocampal and amygdalar volumes (left and right hemisphere), as well as estimated total intracranial volume, from the aseg.stats output files in FreeSurfer (https: / / surfer.nmr. mgh.harvard.edu/fswiki/asegstats2table). We used grouplevel means, standard deviations and histogram plots to identify statistical outliers (i.e., if their volume was measured to be greater than 2.698 standard deviations [SDs] from the global mean) and to confirm the normal distribution of volumetric data. One control participant was identified as an outlier for the estimate of right amygdala volume. In this case, we visually reinspected the images using Freeview (v2.0) in FreeSurfer to confirm segmentation accuracy, and the data were retained in the analysis.

We calculated the relative volume ratio for the anatomic regions of interest as the volume in native space $(\mathrm{mL})$ divided by the total intracranial volume $(\mathrm{L})$. We performed MRI assessments when patients were able to stand still (i.e., they did not have high psychomotor agitation or disorganized behaviour and could tolerate the MRI procedure). The scan was taken within 2 weeks of the initial assessment. 


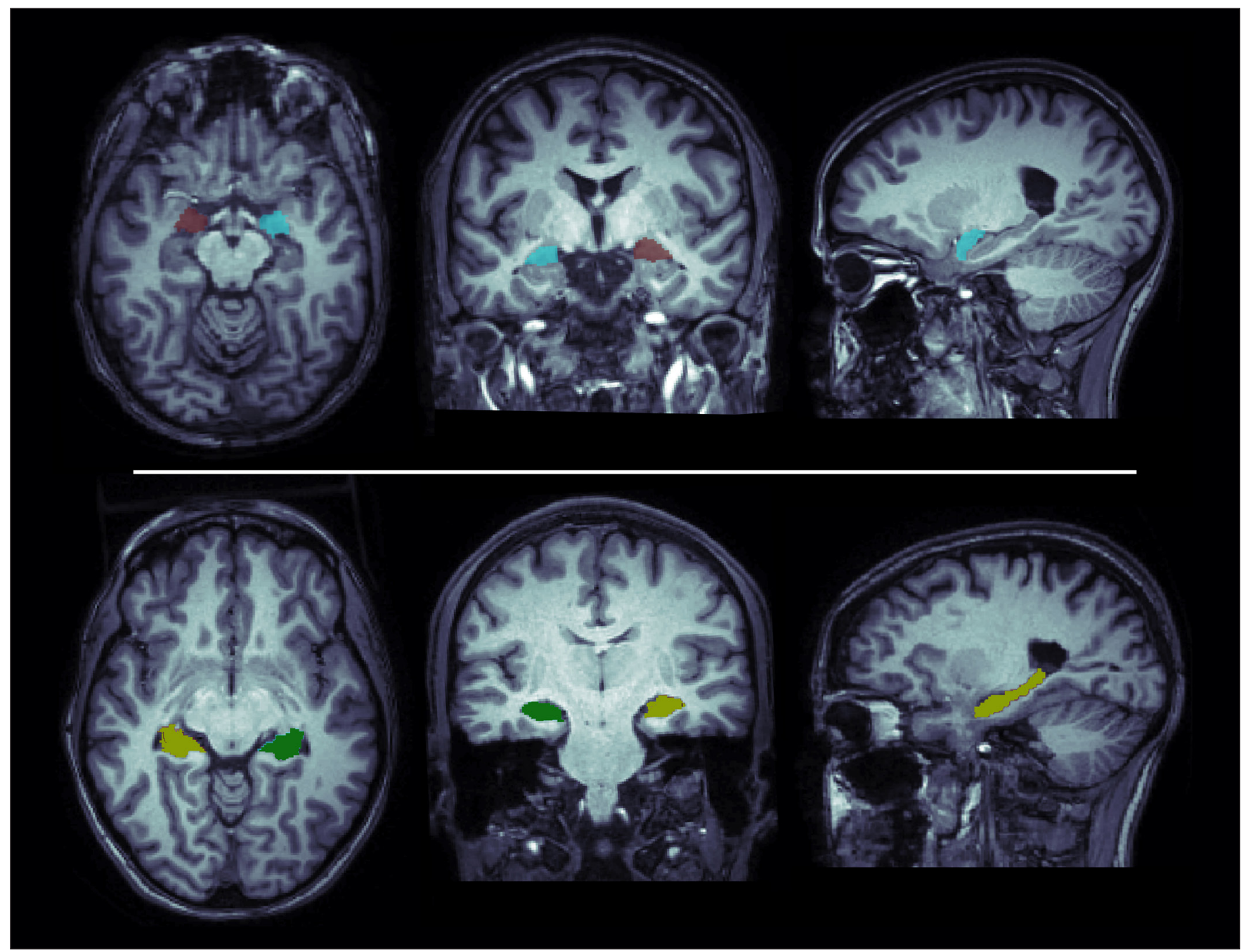

Figure 1: Illustrations of the amygdala (top row) and the hippocampus (bottom row) as segmented by FreeSurfer in a control participant. Segmentations are overlaid on the participant's anatomic MRI.

\section{Statistical analysis}

We assessed data distribution normality using the KolmogorovSmirnov test. Only age, duration of untreated psychosis and tobacco use were non-Gaussian variables. We performed univariate analyses to evaluate differences between the patients who completed the entire follow-up and those who did not. We also performed univariate analyses to assess between-group differences (patients and healthy controls), and Bonferroni correction on brainvolume comparisons.

To determine predictors of negative symptoms at 1-year follow-up, we first conducted univariate analyses that included sociodemographic and baseline clinical and neurobiological variables that were potentially associated with scores on the PANSS negative subscale at 1-year followup: sex, age, duration of untreated psychosis, baseline CDSS score, PANSS positive subscale score, benzodiazepine treatment, left and right amygdala relative volumes, left and right hippocampal relative volumes and serum
BDNF levels. We also accounted for antipsychotic treatment at follow-up as a possible confounding factor.

Next, we developed a linear regression model using the PANSS negative subscale at 1-year follow-up as the dependent variable. Independent variables were all baseline variables (age, sex, cannabis use, tobacco use, duration of untreated psychosis, PANSS negative subscale, PANSS positive subscale, CDSS, GAF, BDNF, left and right amygdala relative volume, left and right hippocampus relative volume, and benzodiazepine treatment) and chlorpromazine equivalents at 1 year follow-up. We also performed an exploratory analysis to assess predictors of the PANSS negative subscale items using the same methodology.

To determine predictors of persistent negative symptoms at 1-year follow-up, we used the same methodology, except that we used a logistic regression model with persistent negative symptoms (yes/no) as the dependent variable.

All statistical analyses were performed in SPSS Statistics for Windows, version 20 (IBM Corp.); $p$ values $\leq 0.05$ were considered statistically significant. 


\section{Results}

\section{Characteristics of patients and healthy controls}

A total of 50 patients were initially included in the study. None of the patients scored 6 or higher on the CDSS at baseline or at follow-up. More than half $(56 \%)$ of the patients were male. The median age was 26 years (interquartile range 24-30.25 years). Most of the patients were being treated with benzodiazepines at inclusion (86\%).

Eleven patients were lost to follow-up. However, we found no statistically significant differences between patients who completed the study and those who did not (Appendix 1, Table S2). At 1-year follow-up, mean ( \pm SD) PANSS scores in patients with first-episode psychosis were as follows: positive subscale $12.69 \pm 7.03$; negative subscale $17.18 \pm 5.89$; general pathology $31.67 \pm 9.26$; and total score $62.51 \pm 19.39$. Of the patient group, $41 \%$ had persistent negative symptoms, and the mean $( \pm$ SD) GAF score was $61.18 \pm 17.68$.

A total of 50 healthy controls were included in this study. More than half $(56 \%)$ were male, and the median age was 26 years (interquartile range 23-30 years). Other clinical characteristics of the sample are shown in Table 1.

Hippocampal and amygdalar absolute volumes (group means \pm SD; patients: left hippocampus $3.88 \pm 0.38 \mathrm{~mL}$, right hippocampus $4.09 \pm 0.45 \mathrm{~mL}$, left amygdala $1.48 \pm 0.24 \mathrm{~mL}$, right amygdala $1.68 \pm 0.22 \mathrm{~mL}$; healthy controls: left hippo- campus $4.17 \pm 0.42 \mathrm{~mL}$, right hippocampus $4.28 \pm 0.45 \mathrm{~mL}$, left amygdala $1.67 \pm 0.20 \mathrm{~mL}$, right amygdala $1.89 \pm 0.25 \mathrm{~mL}$ ) were comparable to those previously reported in similar clinical populations using the same FreeSurfer method. ${ }^{41-44,46,47}$

Univariate analysis (Table 1) showed that patients had significantly smaller relative volumes in the left and right amygdala $(t=-4.26, p=0.001 ; t=-4.32, p=0.001)$ compared to healthy controls. Initially, left and right hippocampal relative volumes were significantly lower in patients compared to healthy controls $(t=-3.63, p=0.047 ; t=-2.13$, $p=0.048$; Appendix 1, Figures S2 and S3), but after Bonferroni correction these findings were no longer significant $(p=0.047$ and $0.048>0.05 / 4=0.0125)$.

Predictors of negative symptoms measured with PANSS at 1-year follow-up

In the univariate analysis (Table 2), PANSS negative subscale scores at 1 year were negatively correlated with baseline serum BDNF levels $(r=-0.4 ; p=0.012)$ and with left amygdala relative volume $(r=-0.51 ; p=0.001)$ in patients with first-episode psychosis. Conversely, PANSS negative subscale scores at 1 year were positively correlated with PANSS negative subscale scores at baseline $(r=0.41 ; p=0.009)$.

In the multivariate analysis, the model that best predicted PANSS negative subscale scores at 1-year follow-up included the following baseline variables: serum BDNF,

Table 1: Sociodemographic, volumetric and clinical characteristics at baseline

\begin{tabular}{|c|c|c|c|c|c|}
\hline Characteristic & $\begin{array}{l}\text { Patients } \\
n=50^{\star}\end{array}$ & $\begin{array}{l}\text { Healthy volunteers } \\
\qquad n=50^{\star}\end{array}$ & Statistical test & $p$ value & Effect size $†$ \\
\hline Age, yr & $26(24-30.25)$ & $26(23-30)$ & $t=992$ & 0.07 & $d=-0.36$ \\
\hline Female & $22(44 \%)$ & $22(44 \%)$ & $\chi^{2}=0.16$ & 0.42 & $V=0.08$ \\
\hline \multicolumn{6}{|l|}{ Relative volume, $\mathrm{mL}$} \\
\hline Left hippocampus & $2.64 \pm 0.27$ & $2.77 \pm 0.35$ & $t=-3.63$ & 0.047 & $d=-0.42$ \\
\hline Right hippocampus & $2.78 \pm 0.31$ & $2.84 \pm 0.36$ & $t=-2.13$ & 0.048 & $d=-0.18$ \\
\hline Left amygdala & $1.01 \pm 0.14$ & $1.11 \pm 0.14$ & $t=-4.26$ & 0.001 & $d=-0.71$ \\
\hline Right amygdala & $1.14 \pm 0.15$ & $1.24 \pm 0.14$ & $t=-4.32$ & 0.001 & $d=-0.69$ \\
\hline Duration of untreated psychosis, $d$ & $31(8-115)$ & - & - & - & - \\
\hline Cannabis user & $29(58 \%)$ & 一 & 一 & - & 一 \\
\hline Tobacco use, cigarettes/d & $4.5(0-14)$ & 一 & 一 & 一 & 一 \\
\hline \multicolumn{6}{|l|}{ Diagnosis } \\
\hline Schizophreniform disorder & $18(36 \%)$ & - & - & - & - \\
\hline Psychosis not otherwise specified & $28(56 \%)$ & - & - & - & - \\
\hline Brief psychotic disorder & $4(8 \%)$ & - & - & - & - \\
\hline Benzodiazepine treatment & $43(86 \%)$ & - & - & - & - \\
\hline BDNF, pg/mL & $45.27 \pm 27.14$ & - & - & - & - \\
\hline PANSS positive subscale score & $24.88 \pm 6.74$ & - & - & - & - \\
\hline PANSS negative subscale score & $16.86 \pm 6.65$ & - & - & - & - \\
\hline PANSS general pathology score & $43.68 \pm 8.27$ & - & - & - & - \\
\hline PANSS total score & $85.24 \pm 15.76$ & - & - & - & - \\
\hline CDSS score & $1.22 \pm 2.02$ & 一 & - & - & 一 \\
\hline GAF score & $29.7 \pm 8.89$ & 一 & 一 & - & 一 \\
\hline \multicolumn{6}{|c|}{$\begin{array}{l}\text { BDNF = brain-derived neurotrophic factor, CDSS }=\text { Calgary Depression Scale for Schizc } \\
\text { Negative Syndrome Scale. } \\
\text { *Values are given as mean } \pm \text { standard deviation, } n(\%) \text { or median (interquartile range). } \\
\text { †Cohen } d \text { or Cramer } V .\end{array}$} \\
\hline
\end{tabular}


Table 2: Association of sociodemographic, volumetric and clinical characterics with PANSS negative score and persistent negative symptoms at 1-year follow-up: univariate analysis

\begin{tabular}{|c|c|c|c|c|c|c|}
\hline Characteristic & $\begin{array}{l}\text { PANSS negative } \\
\text { score at } 1 \text { year }\end{array}$ & $p$ value & Effect size* & $\begin{array}{l}\text { Persistent negative } \\
\text { symptoms at } 1 \text { year }\end{array}$ & $p$ value & Effect size $^{*}$ \\
\hline Age & $r=-0.07$ & 0.69 & - & $t=0.76$ & 0.45 & $d=0.08$ \\
\hline Sex & $t=1.95$ & 0.06 & $d=0.64$ & $\chi^{2}=0.24$ & 0.81 & $V=0.24$ \\
\hline Male & $18.58 \pm 5.68$ & - & - & $68.8 \%$ & - & - \\
\hline Female & $14.93 \pm 5.71$ & - & - & $31.2 \%$ & - & - \\
\hline \multicolumn{7}{|l|}{ Relative volume } \\
\hline Left hippocampus & $r=-0.25$ & 0.12 & - & $t=1.01$ & 0.32 & $d=0.37$ \\
\hline Right hippocampus & $r=-0.21$ & 0.19 & - & $t=1.21$ & 0.23 & $d=0.44$ \\
\hline Left amygdala & $r=-0.51$ & 0.001 & - & $t=2.47$ & 0.018 & $d=0.91$ \\
\hline Right amygdala & $r=-0.31$ & 0.05 & - & $t=1.83$ & 0.08 & $d=0.57$ \\
\hline Duration of untreated psychosis & $r=0.29$ & 0.07 & - & $t=-1.53$ & 0.14 & $d=-0.48$ \\
\hline Cannabis use & $t=-1.2$ & 0.32 & $d=-0.32$ & $\chi^{2}=-0.51$ & 0.62 & $V=0.21$ \\
\hline Non-user & $16.21 \pm 5.5$ & - & - & $43.8 \%$ & - & - \\
\hline User & $18.1 \pm 6.25$ & - & - & $56.2 \%$ & - & - \\
\hline Tobacco use & $r=-0.27$ & 0.10 & - & $t=1.49$ & 0.14 & $d=0.46$ \\
\hline Chlorpromazine equivalent at 1 year & $r=0.09$ & 0.59 & - & $t=0.34$ & 0.74 & $d=0.11$ \\
\hline Benzodiazepine treatment & $t=-1.61$ & 0.12 & $d=-0.64$ & $\chi^{2}=1.93$ & 0.17 & $V=0.41$ \\
\hline BDNF, baseline & $r=-0.4$ & 0.012 & - & $t=2.93$ & 0.006 & $d=1.01$ \\
\hline PANSS negative subscale score, baseline & $r=0.41$ & 0.009 & - & - & & \\
\hline PANSS positive subscale score, baseline & $r=-0.27$ & 0.09 & - & $t=1.23$ & 0.23 & $d=0.39$ \\
\hline CDSS score, baseline & $r=-0.16$ & 0.34 & - & $t=0.45$ & 0.66 & $d=0.14$ \\
\hline GAF score, baseline & $r=-0.26$ & 0.12 & - & $t=2.12$ & 0.07 & $d=0.72$ \\
\hline
\end{tabular}

PANSS negative subscale scores and left amygdala relative volume, with an $R^{2}$ of 0.478 . This finding confirmed that lower baseline serum BDNF level (95\% confidence interval [CI] -0.12 to $-0.02 ; p=0.011)$, smaller left amygdala relative volume (95\% CI -33.14 to $-9.03 ; p=0.001)$, and higher baseline PANSS negative subscale score (95\% CI 0.04 to $0.48 ; p=$ $0.021)$ were associated with higher PANSS negative subscale scores at 1-year follow-up in patients with first-episode psychosis (Table 3 and Figure 2). When we conducted the different models adding 1 variable at a time, BDNF explained $15.8 \%$ of the variance, baseline PANSS negative subscale score explained $13.2 \%$ and left amygdala relative volume explained $18.8 \%$. We also performed a robust regression to confirm that these associations held (with PANSS negative subscale score at 1-year follow-up as a dependent variable and BDNF level, left amygdala relative volume and baseline PANSS negative subscale score as independent variables; Appendix 1, Table S4).

We performed an exploratory univariate analysis to assess predictors of PANSS negative subscale subitems at 1-year follow-up (Appendix 1, Table S3). In a multivariate analysis, baseline PANSS negative subscale score was a significant predictor of those subitems. Baseline CDSS score was a significant predictor of N2 (emotional withdrawal; 95\% CI = 0.02 to $0.26, p=0.025$ ) and sex was a significant predictor of $\mathrm{N} 7$ (stereotyped thinking; $95 \% \mathrm{CI}=-1.87$ to $-0.56, p=0.001$ ).

\begin{tabular}{|c|c|c|c|}
\hline Predictor & $R^{2}$ & $\mathrm{~B}(95 \% \mathrm{Cl})$ & $p$ value \\
\hline Model 1 & 0.253 & & $<0.001$ \\
\hline $\begin{array}{l}\text { Relative left amygdala } \\
\text { volume, } \mathrm{mL}\end{array}$ & & $\begin{array}{c}-24.14(-37.98 \text { to } \\
-10.31)\end{array}$ & 0.001 \\
\hline Model 2 & 0.391 & & $<0.001$ \\
\hline $\begin{array}{l}\text { Relative left amygdala } \\
\text { volume, } \mathrm{mL}\end{array}$ & & $\begin{array}{c}-23.2(-35.89 \text { to } \\
-10.51)\end{array}$ & 0.001 \\
\hline BDNF at baseline & & $\begin{array}{c}-0.08(-0.13 \text { to } \\
-0.02)\end{array}$ & 0.007 \\
\hline Model 3 & 0.478 & & $<0.001$ \\
\hline $\begin{array}{l}\text { Relative left amygdala } \\
\text { volume, } \mathrm{mL}\end{array}$ & & $\begin{array}{c}-21.09(-33.14 \text { to } \\
-9.03)\end{array}$ & 0.001 \\
\hline BDNF at baseline & & $\begin{array}{c}-0.07(-0.12 \text { to } \\
-0.02)\end{array}$ & 0.011 \\
\hline $\begin{array}{l}\text { PANSS negative subscale } \\
\text { score at baseline }\end{array}$ & & 0.26 (0.04 to 0.48$)$ & 0.021 \\
\hline
\end{tabular}

Predictors of persistent negative symptoms at 1-year follow-up

In the univariate analysis (Table 2), persistent negative symptoms at 1 year were significantly associated with baseline serum BDNF levels $(t=2.93 ; p=0.006)$ and left 

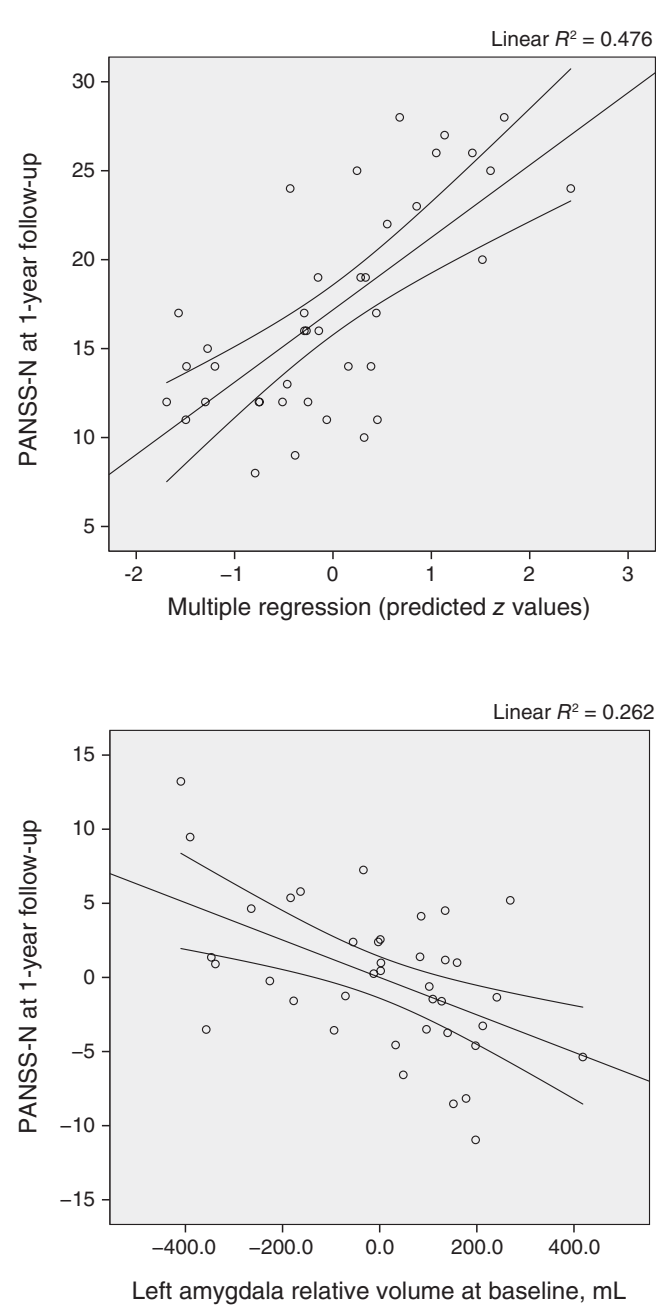
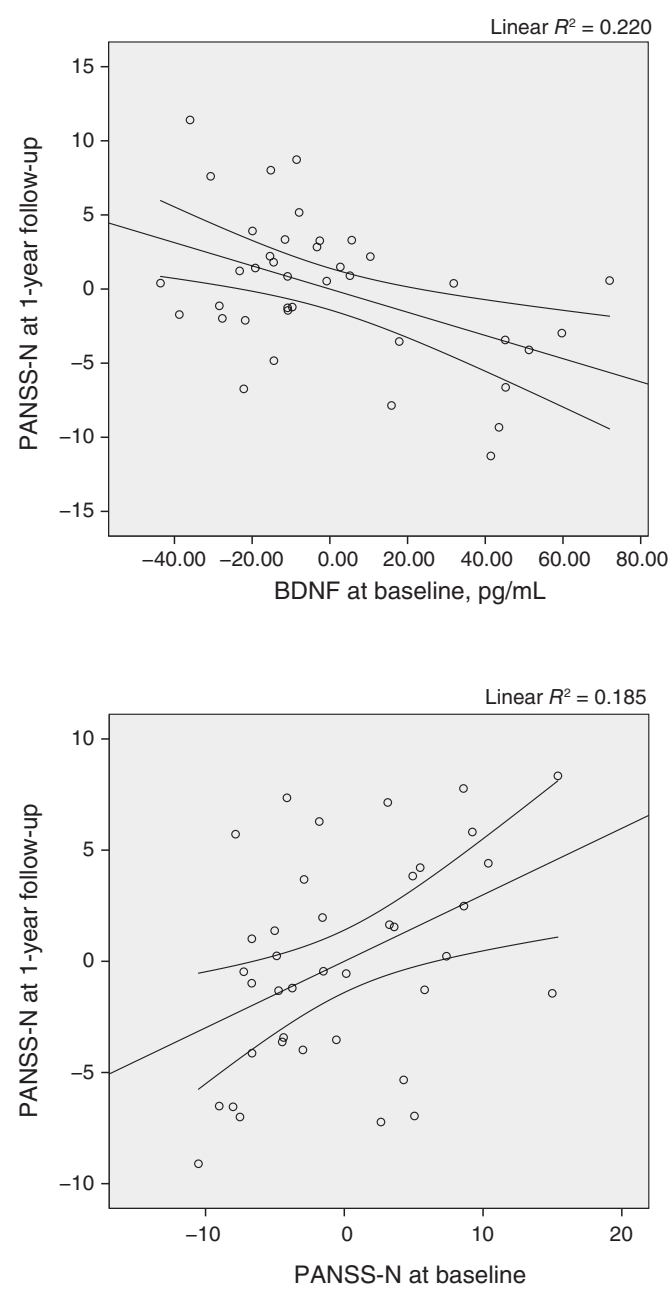

Figure 2: Correlations between PANSS-N scores at 1-year follow-up and BDNF levels, left amygdala relative volume and PANSS-N scores at baseline. BDNF = brain-derived neurotrophic factor; PANSS-N = Positive and Negative Syndrome Scale, negative subscale.

amygdala relative volume $(t=2.47 ; p=0.018)$ in patients with first-episode psychosis (Appendix 1, Figures S4 and S5).

Based on the findings of the multivariate analysis, the final model included 2 baseline variables: serum BDNF levels and left amygdala relative volume $\left(R^{2}=0.533\right)$. These results further supported the association of lower baseline serum BDNF levels (OR 0.93, 95\% CI 0.88 to 0.98 ; $p=0.012$ ) and smaller left amygdala relative volume (OR $0.99,95 \%$ CI 0.98 to $0.99 ; p=0.010$ ) with the presence of persistent negative symptoms at 1 year in patients with first-episode psychosis (Table 4). We repeated this analysis including chlorpromazine equivalents to control for antipsychotic adverse effects and found no significant differences (Appendix 1, Table S4). When we conducted the different models adding 2 variables each time, BDNF explained $27 \%$ of the variance and left amygdala relative volume explained $22.7 \%$ of the variance.

\section{Discussion}

In this study, we found that lower serum BDNF levels, smaller left amygdala relative volume and more negative symptomatology at baseline predicted more negative symptoms (PANSS negative subscale) at 1-year follow-up. In addition, lower baseline serum BDNF levels and a smaller left amygdala relative volume were predictors of persistent negative symptoms at 1 year. Finally, compared with healthy controls, patients with first-episode psychosis had smaller relative amygdalar volumes and a trend toward smaller hippocampal volumes at baseline.

In our cohort, lower baseline serum BDNF levels predicted higher negative symptoms at 1 year, measured using the PANSS negative symptom subscale or persistent negative symptoms. These findings were consistent with previous reports, which have also found that lower serum BDNF levels 
Table 4: Persistent negative symptoms at 1-year follow-up: logistic regression model

\begin{tabular}{|c|c|c|c|c|c|}
\hline Predictor & -2 log-likelihood & Nagelkerke $R^{2}$ & $\chi^{2}$ & OR $(95 \% \mathrm{Cl})$ & $p$ value \\
\hline Step 1 & 44.09 & 0.27 & $\chi^{2}{ }_{1}=8.71$ & & 0.06 \\
\hline BDNF at baseline & & & & 0.96 (0.93 to 0.99$)$ & 0.018 \\
\hline Step 2 & 33.16 & 0.53 & $\chi_{2}^{2}=19.62$ & & \\
\hline Left amygdala relative volume & & & & 0.99 (0.98 to 0.99$)$ & 0.010 \\
\hline BDNF at baseline & & & & 0.93 (0.88 to 0.98$)$ & 0.012 \\
\hline
\end{tabular}

are associated with negative symptoms in patients with firstepisode psychosis ${ }^{48}$ and schizophrenia. ${ }^{49,50}$ Our results were also in line with those of a study that found more severe negative symptoms in patients with first-episode psychosis who were carriers of the Met allele of the BDNF Val66Met polymorphism, a variant that has been associated with decreased BDNF activity. ${ }^{51}$ Nonetheless, a meta-analysis concluded that decreased peripheral BDNF levels did not appear to be associated with the severity of positive or negative symptoms. ${ }^{52}$ However, it is important to note that the association between BDNF levels and negative symptoms in that meta-analysis was assessed cross-sectionally and did not take into account possible confounders, such as secondary sources of negative symptoms.

The findings of this study were in agreement with animal studies showing an influence of BDNF on dopamine release in the mesolimbic dopamine pathway, ${ }^{53}$ which in turn is associated with improved motivational behaviours. ${ }^{54}$ However, the design of the present study did not allow us to test the mechanisms underlying the association between BDNF and negative symptoms.

Left amygdalar relative volume at baseline predicted both negative symptoms (PANSS negative subscale) and persistent negative symptoms at 1-year follow-up. Previous studies have shown that structural abnormalities in the amygdalarhippocampal complex are associated with negative symptoms in patients with schizophrenia and first-episode psychosis. ${ }^{10}$ One study found that patients with first-episode psychosis who presented with persistent negative symptoms had smaller left amygdalar and right hippocampal volumes than patients without persistent negative symptoms. ${ }^{26}$ Furthermore, several studies have found an association between functional activation patterns in the amygdala and the severity of affective flattening in patients with schizophrenia. 55,56

The amygdala plays a key role in emotion and motivational behaviours. ${ }^{57}$ Alterations in its structure might contribute to the differential expression of negative symptoms. The replicated findings regarding the association between negative symptoms and left amygdala volume in first-episode psychosis ${ }^{26-28}$ suggest that the lateralization of amygdala volume differences in patients with first-episode psychosis may be an early predictor of negative symptomatology. This finding would be in line with those of some meta-analyses, which reported that amygdala activations are lateralized to the left, particularly for negative symptoms..$^{58,59}$
Nevertheless, we found no association or mediation effect between negative symptoms and amygdala volume or BDNF levels, as seen in the mediation analysis (Appendix 1, Figure S6). This would suggest that the mechanism by which negative symptoms develop in the case of the amygdala are independent of BDNF levels. As we commented above, BDNF may be associated with negative symptoms through a functional process by increasing dopamine activity in the mesolimbic pathway. ${ }^{53}$

This mechanism could also explain the lack of a significant association between hippocampal volumes and negative symptoms that one would expect, given the known association between hippocampus and BDNF, and given previous findings. Furthermore, it could also be that in previous studies hippocampal volume was not directly associated with negative symptoms, but rather with other symptoms commonly associated with them, such as cognitive symptoms. ${ }^{60}$

In our exploratory analyses of the subitems of the PANSS negative subscale, only the total baseline PANSS negative subscale score predicted PANSS negative subscale subitems at follow-up (except for emotional withdrawal and stereotyped thinking, which were predicted by baseline CDSS scores and sex, respectively). Patients with more symptoms of depression at baseline had higher emotional withdrawal scores at 1-year follow-up. Although it would be reasonable to assume that higher emotional withdrawal scores were attributable to an affective disorder, it is important to note that no patients scored 6 or higher on the CDSS at baseline or follow-up. Consequently, this finding for emotional withdrawal could have been because of difficulties in differentiating between depressive and negative symptoms in patients with psychosis, and particularly in those with first-episode psychosis. On the other hand, the fact that none of the other variables were significant predictors of the PANSS negative subscale subitems could have been secondary to the sample size. However, another explanation could be that the total PANSS negative subscale score measures something different than the sum of the subitems.

The variables that predicted negative symptoms on the PANSS negative subscale and persistent negative symptoms at 1 year were highly consistent. This finding was not surprising, given that baseline PANSS negative subscale scores were among the main predictors of PANSS negative subscale scores at 1 year. These findings were consistent with previous reports, which have found that negative symptoms tend to be persistent if they are already present at illness onset, even though their severity may fluctuate over time. ${ }^{10}$ 
With this multidimensional approach, we predicted $47.8 \%$ of the variance of negative symptomatology measured with the PANSS negative subscale at 1-year follow-up and $53.3 \%$ of the presence of persistent negative symptoms. These findings may help guide clinicians from illness onset (e.g., applying more intensive treatments for negative symptoms in patients who are more vulnerable to severe negative symptoms or persistent negative symptoms in the long term). Among such treatments, ${ }^{61}$ physical activity and add-on antidepressants should be considered, because they have been shown to have beneficial effects on negative symptoms ${ }^{62}$ and to increase BDNF levels. ${ }^{63}$ However, future studies should be carried out to test whether an increase in BDNF levels mediates the improvement of negative symptoms with these treatments. Furthermore, the inclusion of other variables should be considered, such has whole-brain gyrification, $^{64}$ to predict negative symptoms at follow-up even more accurately.

Finally, this study provides additional evidence to the replicated finding that patients with first-episode psychosis have smaller amygdala volumes than healthy controls. ${ }^{65}$ However, a high proportion of our patients were cannabis users, and that could have affected our results. ${ }^{66}$

\section{Limitations}

This study had several limitations. The first is inherent to all longitudinal studies: not all of the participants completed the entire follow-up. Nonetheless, we found no differences in baseline characteristics between completers and noncompleters, indicating that bias was unlikely. In addition, we did not use a scale to assess extrapyramidal symptoms, although we did take into account antipsychotic adverse effects by including chlorpromazine equivalents in the analyses. Finally, we were unable to assess negative symptoms and their dimensions with next-generation scales, which were not available when the study was initiated.

Despite these limitations, this study had several important strengths, most notably the large sample of antipsychotic-naive patients with first-episode psychosis, a patient profile that is particularly difficult to recruit. Another strength was the long follow-up period (1 year). Finally, the innovative, multidimensional baseline assessment used in this study further strengthened our findings. We included numerous parameters (clinical, neuroimaging and laboratory assessment) to build a robust model for predicting negative symptoms.

\section{Conclusion}

Our findings show that several baseline parameters - low serum BDNF levels, decreased left amygdalar relative volume and more negative symptomatology - were predictive of negative symptoms at 1-year follow-up on the PANSS negative subscale (after taking into account secondary sources). Two of those baseline variables - low serum BDNF and a smaller left amygdalar relative volume - were predictive of persistent negative symptoms at 1 year. These findings are highly relevant, because they provide key data to help guide clinicians in developing individualized treatment plans for patients with early-stage disease. However, future studies are needed to replicate these findings and include the different dimensions of negative symptoms, as well as motivation and pleasure and emotional expressivity factors with new generation-scales. ${ }^{67}$

Acknowledgements: The authors thank the patients and volunteers who made this study possible. They also thank Bradley Londres for professional English-language editing.

Affiliations: From the Institut de Neuropsiquiatria i Adiccions (INAD), Parc de Salut Mar, Barcelona, Spain (Toll, Bergé, Canosa, Legido, Pérez-Solà, Mané); the Fundació Hospital del Mar Medical Research Institute (IMIM), Barcelona, Spain (Toll, Bergé, Duran, Canosa, Legido, Pérez-Solà, Mané); the Centro de Investigación Biomédica en Red, Área de Salud Mental (CIBERSAM), Spain (Toll, Blanco-Hinojo, Bergé, Canosa, Pérez-Solà, Mané); the Department of Psychiatry and Forensic Medicine, Universitat Autónoma de Barcelona (UAB), Barcelona, Spain (Toll); the MRI Research Unit, Department of Radiology, Parc de Salut Mar, Barcelona, Spain (Blanco-Hinojo); the Pharmacology Unit, Department of Clinical Fundamentals, Faculty of Medicine, Barcelona University, Barcelona, Spain (Marmol); and the Department of Psychiatry and Behavioral and Clinical Neuroscience Institute, University of Cambridge, United Kingdom (Fernández -Egea).

Competing interests: None declared.

Contributors: A. Toll and A. Mané designed the study. I. Canosa acquired the data, which L. Blanco-Hinojo, D. Bergé, X. Duran, T. Legido, F. Marmol, V. Pérez-Solà and E. Fernández-Egea analyzed. A. Toll wrote the article, which all other authors reviewed. All authors approved the final version to be published and can certify that no other individuals not listed as authors have made substantial contributions to the paper.

Content licence: This is an Open Access article distributed in accordance with the terms of the Creative Commons Attribution (CC BY-NC-ND 4.0) licence, which permits use, distribution and reproduction in any medium, provided that the original publication is properly cited, the use is noncommercial (i.e., research or educational use), and no modifications or adaptations are made. See: https://creativecommons.org/licenses/by-nc-nd/4.0/

\section{References}

1. Mas S, Boloc D, Rodríguez N, et al. Examining gene-environment interactions using aggregate scores in a first-episode psychosis cohort. Schizophr Bull 2020;46:1019-25.

2. GBD 2016 Disease and Injury Incidence and Prevalence Collaborators. Global, regional, and national incidence, prevalence, and years lived with disability for 328 diseases and injuries for 195 countries, 1990-2016: a systematic analysis for the Global Burden of Disease Study 2016. Lancet 2017;390:1211-59.

3. Harvey PD, Strassnig MT, Silberstein J. Prediction of disability in schizophrenia: symptoms, cognition, and self-assessment. J Exp Psychopathol 2019;10:204380871986569.

4. Fusar-Poli P, McGorry PD, Kane JM. Improving outcomes of firstepisode psychosis: an overview. World Psychiatry 2017;16:251-65.

5. Carvalho AF, Solmi M, Sanches M, et al. Evidence-based umbrella review of 162 peripheral biomarkers for major mental disorders. Transl Psychiatry 2020;10:152.

6. Fusar-Poli P, Hijazi Z, Stahl D, et al. The science of prognosis in psychiatry: a review. JAMA Psychiatry 2018;75:1289-97.

7. Ross CA, Margolis RL, Reading SA, et al. Neurobiology of schizophrenia. Neuron 2006;52:139-53. 
8. Harvey PD, Strassnig M. Predicting the severity of everyday functional disability in people with schizophrenia: cognitive deficits, functional capacity, symptoms, and health status. World Psychiatry 2012;11:73-9.

9. Galderisi S, Rossi A, Rocca P, et al. The influence of illness-related variables, personal resources and context-related factors on reallife functioning of people with schizophrenia. World Psychiatry 2014;13:275-87.

10. Hovington CL, Lepage $\mathrm{M}$. Neurocognition and neuroimaging of persistent negative symptoms of schizophrenia. Expert Rev Neurother 2012;12:53-69.

11. Mezquida G, Cabrera B, Bioque M, et al. The course of negative symptoms in first-episode schizophrenia and its predictors: a prospective two-year follow-up study. Schizophr Res 2017;189:84-90.

12. Kirschner M, Aleman A, Kaiser S. Secondary negative symptoms-a review of mechanisms, assessment and treatment. Schizophr Res 2017;186:29-38.

13. Carpenter WT, Heinrichs DW, Wagman AM. Deficit and nondeficit forms of schizophrenia: the concept. Am J Psychiatry 1988;145:578-83.

14. Buchanan RW. Persistent negative symptoms in schizophrenia: an overview. Schizophr Bull 2007;33:1013-22.

15. Ochoa S, Usall J, Cobo J, et al. Gender differences in schizophrenia and first-episode psychosis: a comprehensive literature review. Schizophr Res Treatment 2012;2012:916198.

16. Morgan VA, Castle DJ, Jablensky AV. Do women express and experience psychosis differently from men? Epidemiological evidence from the Australian National Study of Low Prevalence (Psychotic) Disorders. Aust N Z J Psychiatry 2008;42:74-82.

17. Kao YC, Liu YP. Effects of age of onset on clinical characteristics in schizophrenia spectrum disorders. BMC Psychiatry 2010;10:63.

18. Schultz SK, Ho BC, Andreasen NC. Clinical features characterizing young-onset and intermediate-onset schizophrenia. J Neuropsychiatry Clin Neurosci 2000;12:502-5.

19. Boonstra N, Klaassen R, Sytema S, et al. Duration of untreated psychosis and negative symptoms-a systematic review and metaanalysis of individual patient data. Schizophr Res 2012;142:12-9.

20. Jabar LSA, Sørensen HJ, Nordentoft M, et al. Associations between duration of untreated psychosis and domains of positive and negative symptoms persist after 10 years of follow-up: a secondary analysis from the OPUS trial. Schizophr Res 2020;30:30567-3.

21. Immonen J, Jääskeläinen $\mathrm{E}$, Korpela $\mathrm{H}$, et al. Age at onset and the outcomes of schizophrenia: a systematic review and meta-analysis. Early Interv Psychiatry 2017;11:453-60.

22. Sabe M, Zhao N, Kaiser S. Cannabis, nicotine and the negative symptoms of schizophrenia: systematic review and meta-analysis of observational studies. Neurosci Biobehav Rev 2020;116:415-25.

23. Shenton ME, Whitford TJ, Kubicki M. Structural neuroimaging in schizophrenia: from methods to insights to treatments. Dialogues Clin Neurosci 2010;12:317-32.

24. Rajarethinam R, DeQuardo JR, Miedler J, et al. Hippocampus and amygdala in schizophrenia: assessment of the relationship of neuroanatomy to psychopathology. Psychiatry Res 2001;108:79-87.

25. Koutsouleris N, Gaser C, Jäger M, et al. Structural correlates of psychopathological symptom dimensions in schizophrenia: a voxel-based morphometric study. Neuroimage 2008;39:1600-12.

26. Makowski C, Bodnar M, Shenker JJ, et al. Linking persistent negative symptoms to amygdala-hippocampus structure in first-episode psychosis. Transl Psychiatry 2017;7:e1195.

27. Honea R, Crow TJ, Passingham D, et al. Regional deficits in brain volume in schizophrenia: a meta-analysis of voxel-based morphometry studies. Am J Psychiatry 2005;162:2233-45.

28. Bois C, Levita L, Ripp I, et al. Hippocampal, amygdala and nucleus accumbens volume in first-episode schizophrenia patients and individuals at high familial risk: a cross-sectional comparison. Schizophr Res 2015;165:45-51.
29. Numakawa T, Odaka H, Adachi N. Derived neurotrophin factor in the neurogenesis and neuronal function, and its involvement in the pathophysiology of brain diseases. Int J Mol Sci 2018;19:3650.

30. Green MJ, Matheson SL, Shepherd A, et al. Brain-derived neurotrophic factor levels in schizophrenia: a systematic review with meta-analysis. Mol Psychiatry 2011;16:960-72.

31. Toll A, Mané A. Brain-derived neurotrophic factor levels in first episode of psychosis: a systematic review. World J Psychiatry 2015;5:154-9.

32. Niitsu $\mathrm{T}$, Ishima $\mathrm{T}$, Yoshida $\mathrm{T}$, et al. A positive correlation between serum levels of mature brain-derived neurotrophic factor and negative symptoms in schizophrenia. Psychiatry Res 2014;215:268-73.

33. Bergé $\mathrm{D}$, Mané $\mathrm{A}$, Salgado $\mathrm{P}$, et al. Predictors of relapse and functioning in first-episode psychosis: a two-year follow-up study. Psychiatr Serv 2016;67:227-33

34. Strauss GP, Cohen AS. A transdiagnostic review of negative symptom phenomenology and etiology. Schizophr Bull 2017;43:712-9.

35. Kay SR. Positive-negative symptom assessment in schizophrenia: psychometric issues and scale comparison. Psychiatr Q 1990;61:163-78.

36. Aas IH. Guidelines for rating Global Assessment of Functioning (GAF). Ann Gen Psychiatry 2011;10:2.

37. Addington D, Addington J, Schissel B. A depression rating scale for schizophrenics. Schizophr Res 1990;3:247-51.

38. Gardner DM, Murphy AL, O'Donnell H, et al. International consensus study of antipsychotic dosing. Am J Psychiatry 2010;167:686-93.

39. Galderisi S, Mucci A, Bitter I, et al. Persistent negative symptoms in first episode patients with schizophrenia: results from the European First Episode Schizophrenia Trial. Eur Neuropsychopharmacol 2013;23:196-204.

40. Piccinni A, Marazziti D, Del Debbio A, et al. Diurnal variation of plasma brain-derived neurotrophic factor (BDNF) in humans: an analysis of sex differences. Chronobiol Int 2008;25:819-26.

41. Arnold SJM, Ivleva EI, Gopal TA, et al. Hippocampal volume is reduced in schizophrenia and schizoaffective disorder but not in psychotic bipolar I disorder demonstrated by both manual tracing and automated parcellation (FreeSurfer). Schizophr Bull 2015;41:233-49.

42. Haukvik UK, Hartberg CB, Nerland S, et al. No progressive brain changes during a 1-year follow-up of patients with first-episode psychosis. Psychol Med 2016;46:589-98.

43. Sasabayashi D, Takayanagi $Y$, Takahashi T, et al. Subcortical brain volume abnormalities in individuals with at-risk mental state. Schizophr Bull 2020;46:834-45.

44. van Erp TG, Hibar DP, Rasmussen JM, et al. Subcortical brain volume abnormalities in 2028 individuals with schizophrenia and 2540 healthy controls via the ENIGMA consortium. Mol Psychiatry 2016;21:547-53.

45. Fischl B, Salat DH, Busa E, et al. Whole brain segmentation: automated labeling of neuroanatomical structures in the human brain. Neuron 2002;33:341-55.

46. Pujol N, Mané A, Bergé D, et al. PEPs GROUP. Influence of BDNF and MTHFR polymorphisms on hippocampal volume in first-episode psychosis. Schizophr Res 2020;223:345-52.

47. Szamosi A, Kelemen O, Kéri S. Hippocampal volume and the AKT signaling system in first-episode schizophrenia. J Psychiatr Res 2012. 46:279-84

48. Rizos EN, Michalopoulou PG, Siafakas N, et al. Association of serum brain-derived neurotrophic factor and duration of untreated psychosis in first-episode patients with schizophrenia. Neuropsychobiology 2010;62:87-90.

49. Carlino D, Leone E, Di Cola F, et al. Low serum truncated-BDNF isoform correlates with higher cognitive impairment in schizophrenia. J Psychiatr Res 2011;45:273-9.

50. Chen DC, Wang J, Wang B, et al. Decreased levels of serum brainderived neurotrophic factor in drug-naïve first-episode schizophrenia: relationship to clinical phenotypes. Psychopharmacology (Berl) 2009:207:375-80. 
51. Mezquida G, Penadés R, Cabrera B, et al. Association of the brainderived neurotrophic factor Val66Met polymorphism with negative symptoms severity, but not cognitive function, in first-episode schizophrenia spectrum disorders. Eur Psychiatry 2016;38:61-9.

52. Fernandes BS, Steiner J, Berk M, et al. Peripheral brain-derived neurotrophic factor in schizophrenia and the role of antipsychotics: meta-analysis and implications. Mol Psychiatry 2015;20:1108-19.

53. Narita M, Aoki K, Takagi M, et al. Implication of brain-derived neurotrophic factor in the release of dopamine and dopamine-related behaviors induced by methamphetamine. Neuroscience 2003; 119:767-75.

54. Boekhoudt L, Wijbrans EC, Man JHK, et al. Enhancing excitability of dopamine neurons promotes motivational behaviour through increased action initiation. Eur Neuropsychopharmacol 2018;28:171-84.

55. Lepage M, Sergerie K, Benoit A, et al. Emotional face processing and flat affect in schizophrenia: functional and structural neural. correlates. Psychol Med 2011;41:1833-44.

56. Gur RE, Loughead J, Kohler CG, et al. Limbic activation associated with misidentification of fearful faces and flat affect in schizophrenia. Arch Gen Psychiatry 2007;64:1356-66.

57. Fernando AB, Murray JE, Milton AL. The amygdala: securing pleasure and avoiding pain. Front Behav Neurosci 2013;7:190.

58. Baas D, Aleman A, Kahn R. Lateralization of amygdala activation: a systematic review of functional neuroimaging studies. Brain Res Rev 2004;45:96-10.
59. Wager TD, Phan KL, Liberzon I, et al. Valence, gender, and lateralization of functional brain anatomy in emotion: a meta-analysis of findings from neuroimaging. Neuroimage 2003;19:513-31.

60. Hovington $\mathrm{CL}$, Bodnar $\mathrm{M}$, Joober $\mathrm{R}$, et al. Impairment in verbal memory observed in first episode psychosis patients with persistent negative symptoms. Schizophr Res 2013;147:223-9.

61. Galderisi S, Mucci A, Dollfus S, et al. EPA guidance on assessment of negative symptoms in schizophrenia. Eur Psychiatry 2021;64:e23.

62. Sabe M, Kaiser S, Sentissi O. Physical exercise for negative symptoms of schizophrenia: systematic review of randomized controlled trials and meta-analysis. Gen Hosp Psychiatry 2020;62:13-20.

63. Björkholm C, Monteggia LM. BDNF-a key transducer of antidepressant effects. Neuropharmacology 2016;102:72-9.

64. Hauke DJ, Schmidt A, Studerus E, et al. PRONIA Group. Multimodal prognosis of negative symptom severity in individuals at increased risk of developing psychosis. Transl Psychiatry 2021; 11:312.

65. Watson DR, Bai F, Barrett SL, et al. Structural changes in the hippocampus and amygdala at first episode of psychosis. Brain Imaging Behav 2012;6:49-60.

66. Batalla A, Bhattacharyya S, Yücel M, et al. Structural and functional imaging studies in chronic cannabis users: a systematic review of adolescent and adult findings. PLoS One 2013;8:e55821.

67. Strauss GP, Hong LE, Gold JM, et al. Factor structure of the Brief Negative Symptom Scale. Schizophr Res 2012;142:96-8. 